\title{
ROLE OF HYPOTHERMIA IN CONTEMPORARY MEDICINE
}

\author{
Lukasz Szarpak
}

Department of Emergency Medicine, Medical University of Warsaw, Poland

Disaster Emerg Med J 2017; 2(2): 55-57

In recent years, cooling of the patient after cardiac arrest has become one of the basic procedures during post-resuscitation care, which has been reflected in the guidelines of both the European Resuscitation Council and the American Heart Association. Targeted temperature management (TTM), previously known as therapeutic hypothermia, is a procedure associated with controlled lowering of body temperature to prevent tissue and organ damage and has well-established role in emergency medicine.

Currently, many techniques of lowering body temperature are available, including surface cooling and invasive cooling. In patients with subarachnoid haemorrhage, treatment with intravenous cooling catheters reduces the risk of thrombotic complications such as pulmonary embolism and thrombosis. In a study by Pittl et al., the use of surface and invasive cooling showed comparable effectiveness in lowering the patient temperature, but invasive cooling provided higher temperature stability, although it was also associated with a higher incidence of bleeding. Both cooling techniques were also associated with comparable survival to hospital discharge and comparable neurological status of patients. Regardless of the method of hypothermia induction, continuous measurements of core body temperature of the patient are essential and the temperature of the brain should not differ from the body temperature by more than $0.1-0.2$ degrees [1]. It is recommended that the temperature be measured in at least two different locations, in order to obtain accurate measurement results. Most commonly used are sensors placed in the oesophagus, urinary bladder or pulmonary artery.

In the induction phase, TTM may result in hypovolaemia, increased haematocrit, microcirculation disturbances, arrhythmias and electrolyte abnormal- ities; therefore, the patient's condition should be monitored and all abnormalities corrected. The main adverse effect during TTM induction is shivering. To avoid this complication, magnesium infusion can be used to maintain magnesium level above $2 \mathrm{mmol} / \mathrm{L}$, as well as sedatives and, ultimately, the neuromuscular blocking agents.

Current recommendations of the International Liaison Committee on Resuscitation and (ILCOR) and guidelines of both the European Resuscitation Council [2] and the American Cardiac Society [3] recommend the use of TTM in all comatose patients ( $\leq 8$ pts) who experience return of spontaneous circulation (ROSC) following sudden cardiac arrest. Hypothermia within $32-36^{\circ} \mathrm{C}$ should be maintained for 24 hours and then continued for at least next 24 hours. Polderman [4] in his study on an animal model has shown that the earlier the induction of therapeutic hypothermia, the faster the target temperature is achieved, which translates into good neurological status. However, both randomized trial by Bernard et al. [5] and meta-analysis by Diao et al. [6] have shown that immediate cooling of the patient at the scene of the accident had no effect on survival or improvement in neurological status. As shown in the RINSE-Trial performed by Bernard et al., the use of rapid infusion of cold saline during resuscitation of patients with defibrillation rhythm may reduce the rate of spontaneous cardioversion [7]. The therapeutic window for the use of TTM in patients after ROSC remains unknown. Currently, the protocols are based on the study by Bernard et al. and the Hypothermia after Cardiac Arrest (HACA) group, according to which TTM should be implemented within 6 hours after the return of spontaneous circulation. 
Therapeutic hypothermia has been used both in patients with defibrillation [8] and non-defibrillation [9] rhythms. When compared with normothermia, TTM improves prognosis in adults after spontaneous cardioversion $[2,3]$, but not in outpatient $[10,11]$ or in-hospital [12] paediatric patients with cardiac arrest. Moreover, TTM should also be used in unconscious patients with cardiac arrest complicated by myocardial infarction.

Mild to moderate hypothermia improves neurological prognosis in neonates with hypoxic brain injury [12]. Newborns with moderate to severe hypoxic-ischaemic encephalopathy should be provided with therapeutic hypothermia. Jacobs et al. suggested that TTM should be used in children born before 36 weeks of gestation. There are biological evidences indicating that TTM should be considered as a treatment option in premature infants [13]. Treatment should be in accordance with protocols used in randomized clinical trials: TTM should be initiated within 6 hours after birth and continued up to 72 hours after birth, with heating duration of at least 4 hours. Shankaran et al. have shown that whole-body hypothermia reduces the risk of death and disability in infants with moderate to severe hypoxic-ischaemic encephalopathy [12]. Therapeutic hypothermia is also associated with a decrease in seizures after discharge from intensive care units [14].

Effective control of body temperature is one of the key components of the management of patients requiring intensive neurological care. Diringer et al. have demonstrated that elevated temperature in patients treated in the intensive care unit was associated with prolonged hospital stay, poorer prognosis and increased mortality [15]. Lowering the brain temperature by only $2-3$ degrees in patients with acute cerebral ischaemia significantly reduces brain damage [16]. Therapeutic hypothermia in ischaemic stroke may potentially be effective in reducing brain oedema around the haematoma and improving treatment outcomes [17]. The authors of COOLAID and ICTUS-L studies have shown that TTM is an easy and safe procedure for patients with acute stroke, although associated with adverse effects (such as shivering, pneumonia and hypertension). Researchers involved in the COOLAID Oresund programme have concluded that the use of TTM in intensive care units provides better control of shivering and temperature itself, and that the cooling of unconscious patients with stroke can be used to monitor their neurological status. Abdullah in the pilot study has also demonstrated the effectiveness of TTM in the management of haemorrhagic stroke [18].

Therapeutic hypothermia is used in adult patients after traumatic brain injury, improving neurological status and reducing mortality. Meta-analysis by Crampton et al. [19] indicated that the optimal cooling strategy in traumatic brain injury resulting in best protective effects on brain function should be based on cooling the brain and maintaining hypothermia for 72 hours. Studies have shown that the administration of barbiturates may limit the benefits of TTM. Therapeutic hypothermia can be used in the management of traumatic brain injury in adults; however, it is not recommended for paediatric patients.

In conclusion, TTM is widely used to improve neurological outcomes and survival, but despite proven effectiveness it is still a rarely-used procedure in Poland.

Conflict of interest: None declared.

\section{REFERENCES}

1. Tisherman SA. Hypothermia and injury. Curr Opin Crit Care. 2004; 10(6): 512-519, indexed in Pubmed: 15616395.

2. Nolan JP, Soar J, Cariou A, et al. European Resuscitation Council and European Society of Intensive Care Medicine Guidelines for Post-resuscitation Care 2015: Section 5 of the European Resuscitation Council Guidelines for Resuscitation 2015. Resuscitation. 2015; 95: 202-222, doi: 10.1016/j.resuscitation.2015.07.018, indexed in Pubmed: 26477702.

3. Callaway CW, Donnino MW, Fink EL, et al. Part 8: Post-Cardiac Arrest Care: 2015 American Heart Association Guidelines Update for Cardiopulmonary Resuscitation and Emergency Cardiovascular Care. Circulation. 2015; 132(18 Suppl 2): S465-S482, doi: 10.1161/ CIR.0000000000000262, indexed in Pubmed: 26472996.

4. Polderman KH. Mechanisms of action, physiological effects, and complications of hypothermia. Crit Care Med. 2009; 37(7 Suppl): S186-S202, doi: 10.1097/CCM.0b013e3181aa5241, indexed in Pubmed: 19535947.

5. Bernard SA, Smith K, Cameron P, et al. Rapid Infusion of Cold Hartmanns Investigators. Induction of prehospital therapeutic hypothermia after resuscitation from nonventricular fibrillation cardiac arrest*. Crit Care Med. 2012; 40(3): 747-753, doi: 10.1097/ CCM.0b013e3182377038, indexed in Pubmed: 22020244.

6. Diao M, Huang F, Guan J, et al. Prehospital therapeutic hypothermia after cardiac arrest: a systematic review and meta-analysis of randomized controlled trials. Resuscitation. 2013; 84(8): 1021-1028, doi: 10.1016/j.resuscitation.2013.02.003, indexed in Pubmed: 23454259.

7. Bernard SA, Smith K, Finn J, et al. Induction of Therapeutic Hypothermia During Out-of-Hospital Cardiac Arrest Using a Rapid Infusion of 
Cold Saline: The RINSE Trial (Rapid Infusion of Cold Normal Saline). Circulation. 2016; 134(11): 797-805, doi: 10.1161/CIRCULATIONAHA.116.021989, indexed in Pubmed: 27562972.

8. Bernard SA, Gray TW, Buist MD, et al. Treatment of comatose survivors of out-of-hospital cardiac arrest with induced hypothermia. N Engl J Med. 2002; 346(8): 557-563, doi: 10.1056/NEJMoa003289, indexed in Pubmed: 11856794.

9. Lascarrou JB, Meziani F, Le Gouge A, et al. Clinical Research in Intensive Care and Sepsis (CRICS) Group and HYPERION Study Group. Therapeutic hypothermia after nonshockable cardiac arrest: the HYPERION multicenter, randomized, controlled, assessor-blinded, superiority trial. Scand J Trauma Resusc Emerg Med. 2015; 23: 26, doi: 10.1186/ s13049-015-0103-5, indexed in Pubmed: 25882712.

10. Moler FW, Silverstein FS, Holubkov R, et al. THAPCA Trial Investigators. Therapeutic hypothermia after out-of-hospital cardiac arrest in children. N Engl J Med. 2015; 372(20): 1898-1908, doi: 10.1056/ NEJMoa1411480, indexed in Pubmed: 25913022.

11. Moler FW, Silverstein FS, Holubkov R, et al. THAPCA Trial Investigators. Therapeutic Hypothermia after In-Hospital Cardiac Arrest in Children. N Engl J Med. 2017; 376(4): 318-329, doi: 10.1056/NEJMoa1610493, indexed in Pubmed: 28118559.

12. Pappas A, Shankaran S, McDonald SA, et al. Hypothermia Extended Follow-up Subcommittee of the Eunice Kennedy Shriver NICHD Neonatal Research Network, Extended Hypothermia Subcommittee of the Eunice Kennedy Shriver National Institute of Child Health and Human Development Neonatal Research Network, Eunice Kennedy Shriver National Institute of Child Health and Human Development Neonatal Research Network, Eunice Kennedy Shriver National Institute of Child Health and Human Development Neonatal Research Network, Eunice Kennedy Shriver National Institute of Child Health and Human Development Neonatal Research Network, Eunice Kennedy Shriver National Institute of Child Health and Human Development Neonatal Research Network, Eunice Kennedy Shriver National Institute of Child Health and Human
Development Neonatal Research Network, National Institute of Child Health and Human Development Neonatal Research Network, National Institute of Child Health and Human Development Neonatal Research Network. Whole-body hypothermia for neonates with hypoxic-ischemic encephalopathy. N Engl J Med. 2005; 353(15): 1574-1584, doi: 10.1056/NEJMcps050929, indexed in Pubmed: 16221780.

13. Jacobs SE, Berg M, Hunt R, et al. Cooling for newborns with hypoxic ischaemic encephalopathy. Cochrane Database Syst Rev. 2013; 31(1): CD003311.

14. Ghosh S, Tran L, Shuster JJ, et al. Therapeutic hypothermia for neonatal hypoxic ischemic encephalopathy is associated with short-term reduction of seizures after discharge from the neonatal intensive care unit. Childs Nerv Syst. 2017; 33(2): 329-335, doi: 10.1007/s00381016-3321-x, indexed in Pubmed: 27988879.

15. Diringer MN, Reaven NL, Funk SE, et al. Elevated body temperature independently contributes to increased length of stay in neurologic intensive care unit patients. Crit Care Med. 2004; 32(7): 1489-1495, indexed in Pubmed: 15241093.

16. Weinrauch V, Safar $P$, Tisherman $S$, et al. Beneficial effect of mild hypothermia and detrimental effect of deep hypothermia after cardiac arrest in dogs. Stroke. 1992; 23(10): 1454-1462, indexed in Pubmed: 1412583.

17. Melmed KR, Lyden PD. Meta-Analysis of Pre-Clinical Trials of Therapeutic Hypothermia for Intracerebral Hemorrhage. Ther Hypothermia Temp Manag. 2016 [Epub ahead of print], doi: 10.1089/ther.2016.0033, indexed in Pubmed: 27906602.

18. Abdullah JM, Husin A. Intravascular hypothermia for acute hemorrhagic stroke: a pilot study. Acta Neurochir Suppl. 2011; 111: 421-424, doi: 10.1007/978-3-7091-0693-8_72, indexed in Pubmed: 21725794.

19. Crompton EM, Lubomirova I, Cotlarciuc I, et al. Meta-Analysis of Therapeutic Hypothermia for Traumatic Brain Injury in Adult and Pediatric Patients. Crit Care Med. 2017; 45(4): 575-583, doi: 10.1097/ CCM.0000000000002205, indexed in Pubmed: 27941370. 